\title{
Effective scalar fields in Yang-Mills theories
}

\author{
Henri Verschelde ${ }^{\dagger}$, Valentin I. Zakharov ${ }^{* \ddagger}$ \\ ${ }^{\dagger}$ Ghent University, Department of Physics and Astronomy, \\ Krijgslaan 281 - S9, 9000 Ghent, Belgium. \\ *Institute for Theoretical and Experimental Physics, \\ B. Cheremushkinskaya 25, Moscow, 117218, Russia. \\ Max-Planck-Institut für Physik, \\ Föhringer Ring 6, 80805 München, Germany. \\ E-mail: henri.verschelde@ugent.be, xxz@mppmu.mpg • de
}

\begin{abstract}
Scalar fields play a crucial role in the Standard model. On the other hand, in the weak-coupling regime there is an unsolved problem of the quadratic divergence of scalar masses. Thus, it is natural to turn to composite, or effective scalar fields in the strong-coupling regime. Lattice simulations provide information on the "actually existing" scalar fields in Yang-Mills theories. On the continuum-theory side, dual models predict existence of various low-dimension vacuum defects, including probably scalar particles. We are looking for correspondence between the two frameworks, of lattice phenomenology and dual models, and discuss possible applications to the theory of the Yang-Mills plasma in the deconfining phase. It is not ruled out that the effective scalars play important role in the plasma dynamics.
\end{abstract}

The many faces of $Q C D$

November 1-5, 2010

Gent Belgium

\footnotetext{
* Speaker.
} 


\section{Introduction}

The title of the conference invites us to talk about various facets of QCD. One of the most interesting and, nevertheless, rarely covered aspects of QCD is the story about effective scalar, or Higgs-like particles. We are sure about the fundamental Lagrangian of QCD (which we actually reduce, for our purposes, to its pure gluonic part), and there are no fundamental scalar fields in this Lagrangian. Unlike the theory of weak interactions, where one does introduce Higgs fields. In the case of weak coupling, however, there is the problem of the quadratic divergence in the mass, or in the vacuum expectation value of the Higgs field, $\left\langle|H|^{2}>\sim \Lambda_{U V}^{2}\right.$. The authors of the talk belong to the theorists who consider this divergence a fatal failure of the theory. What we actually need to avoid in the hierarchy problem is

$$
<|H|^{2}>\sim \Lambda_{I R}^{2}
$$

where $\Lambda_{I R}$ is the mass scale associated, probably, with new strong interactions, and the Higgs field would be then a composite particle.

In terms of QCD the question is then, whether there exist effective scalar degrees of freedom such that (1.1) is satisfied with $\Lambda_{I R}=\Lambda_{Q C D}$. The path to scalars is provided by considerations of the confinement mechanism in Yang-Mills theories. In Abelian cases [1] the confinement of electric charges is due to condensation of a magnetically charged scalar field. By analogy, one is tempted to assume that in the non-Abelian case the tension of the string connecting heavy quarks is also of the order

$$
\sigma_{Q \bar{Q}} \sim<\Phi_{M}>^{2} \sim \Lambda_{Q C D}^{2} \quad(\text { expectation })
$$

where $\Phi_{M}$ is a magnetically charged composite field. Although the guess (1.2) looks appealing, there are many questions left. What is the precise meaning of the magnetic degree of freedom, how is magnetic charge is defined, which symmetry is spontaneously broken by the vacuum expectation value $\left\langle\Phi_{M}\right\rangle \neq 0$, and so on.

Magnetic degrees of freedom responsible for the confinement have been searched for on the lattice, for review and further references see, e.g., [2], and there exists extensive lattice phenomenology. The interpretation of the lattice data, however, is not straightforward. First, particles and strings on the lattice are to be described in terms of the quantum geometry which deals with lines and surfaces fluctuating on the size of the lattice spacing $a, a \rightarrow 0$ in the continuum limit, see, e.g., [3]. Moreover, there are also specific lattice-related constructions which have no direct analogy in the continuum limit. Finally, every lattice configuration is to be understood as a measurement of the Yang-Mills fields with resolution of the lattice spacing $a$. While physical matrix elements are immune to the details of the measuring procedure, some other observations, like localization of a fermionic zero mode might depend on the measuring procedure [4], as is common in quantum mechanics. Thus, the translation from the lattice to the continuum-theory language is a long process and we refer the reader to [5] and references therein for further details.

Confinement is a non-perturbative phenomenon and infrared physics is involved. In the field theoretic language the only known non-perturbative configuration is the instanton. The dual formulations, completing the Y-M theories in the infrared, involve extra dimensions and are much richer in topologically stable excitations, for particular examples and references see [6, 7]. 
The talk is, essentially, in three parts. First, we review the lattice data on the scalars. Then, we discuss effective scalar fields within the dual models. Finally, we are looking for applications to the the quark-gluon plasma, (for review of the plasma properties see, e.g., [8]).

\section{Particles and strings on the lattice}

\subsection{Lattice monopoles, or scalars}

Monopoles are identified as certain trajectories in the $4 \mathrm{~d}$ volume of the lattice ${ }^{1}$. The properties of the trajectories can be related to field theoretic quantities within the polymer approach to field theory. One of the simplest things to measure is the total length of the monopole trajectories. It turns out that the total length is described by a two-term expression [9]:

$$
\left(L_{\text {tot }}\right)_{\text {monopoles }}=\left(c_{1} \Lambda_{Q C D}^{3}+c_{2} \Lambda_{Q C D}^{2} a^{-1}\right) V_{\text {tot }},
$$

where $V_{\text {tot }}$ is the total volume of the lattice and the origin of this factor is trivial, $c_{1,2}$ are fitting parameters, for details and further references see Ref. [9]. Note that the term proportional to $a^{-1}$ dominates in the continuum limit. Nevertheless, we keep both terms and for a good reason. Namely, the two terms in (2.1) refer to two different types of trajectories. The first term refers to an infinite cluster, while the second term corresponds to finite, or short clusters. In field theoretic terms, an infinite cluster is a condensate and short clusters are quantum fluctuations. It is worth emphasizing that the emergence of the parameter $\Lambda_{Q C D}$ is a highly non-trivial observation since $\Lambda_{Q C D}$ is related to the lattice spacing by means of the two-loop $\beta$-function. The emergence of $\Lambda_{Q C D}$ in numerical simulations provides a strong support to the idea that lattice monopoles correspond indeed to physical objects.

\subsection{Very dilute monopole condensate}

Eq. (2.1) can be considered as the main message from the lattice to the continuum theory. Indeed, for an elementary scalar one would have

$$
L_{\text {elem }} \sim a^{-3} V_{\text {tot }}, \quad<\left|\Phi_{\text {elem }}\right|^{2}>\sim a^{-2}
$$

and reproduce the standard quadratic divergence which plagues the elementary-scalar theories. In contrast to this, Eq. (2.1) implies for the vacuum expectation of the lattice scalar:

$$
<\left|\Phi_{M}\right|^{2}>\sim \lim _{a \rightarrow 0}\left(a \cdot L_{t o t}\right) \sim c_{2} \Lambda_{Q C D}^{2}
$$

This is an amusing result since (2.3) is exactly what we would like to have for a composite Higgs fields, compare (1.1). However, there are surprises left as we will immediately see.

Indeed, for the classical part of the field, or the monopole condensate application of quantum geometry brings an unexpected result [10]:

$$
<\Phi_{M}>^{2} \sim\left(a \cdot \Lambda_{Q C D}\right) \Lambda_{Q C D}^{2},
$$

\footnotetext{
${ }^{1}$ The name of "monopoles" should not be taken too literally and is used mostly for the historic reasons.
} 
so that the classical part vanishes in the continuum limit $a \rightarrow 0$. The lattice result (2.4) is in sharp contrast with the expectation (1.2). Thus, Eq. (2.4) suggests that confinement is ensured by a condensate which is vanishing in the continuum limit! Nevertheless, at any finite value of the lattice spacing $a$ the monopole models do explain the string tension $\sigma_{Q \bar{Q}}$ in terms of the monopole condensate. The price is that the models assume long-range forces, or massless exchange in the Yang-Mills vacuum. Anyhow, we have to look for a substitution for $\left\langle\Phi_{M}\right\rangle$ in Eq. (1.2). Probably, it is the string density which takes over $\left\langle\Phi_{M}>^{2}\right.$, see below.

\subsection{Magnetic surfaces, or strings}

Probably, the most unexpected observation concerning monopoles is that the monopole trajectories do not percolate in fact through the whole $4 \mathrm{~d}$ volume $V_{\text {tot }}$ but rather lie on $2 \mathrm{~d}$ surfaces which are defined independently. Two-dimensional surfaces correspond to strings and one can say, therefore, that the lattice monopoles live on strings. The absence of the ultraviolet divergence in Eq. (2.3) is just a manifestation of this alignment of the monopole trajectories with the vortices.

In more detail, the total area of the surfaces is empirically given by [11,2]:

$$
(\text { Area })_{\text {tot }} \approx c_{3} \Lambda_{Q C D}^{2} V_{\text {tot }} \equiv \rho_{\text {strings }} V_{\text {tot }} .
$$

The surfaces are closed in the vacuum. The monopoles, in turn, are closed trajectories lying on the surfaces. Small clusters of monopole trajectories cover the surfaces densely while the infinite cluster, still belonging to the surfaces, is dilute and occupies a vanishing part of the total area (2.5). In case of the surfaces one can also distinguish clusters, finite or an infinite. However, unlike the monopole case, the total area (2.5) is dominated by a single, percolating cluster of the vortices (or strings).

From the fact that the area (2.5) scales in physical units, see (2.5), one would conclude that the string tension is of order

$$
T_{\text {magmetic strings }} \sim \Lambda_{Q C D}^{2} .
$$

In the continuum limit, the lattice strings are identified as magnetic strings $[5,6]$ since they are closed in the vacuum and can be open on an external 't Hooft line.

\subsection{Deconfinement phase transition}

At the deconfinement phase transition the monopole trajectories and magnetic surfaces become time oriented, for review and references see [2]. Namely, for the monopole trajectories one can introduce an asymmetry parameter

$$
v=\frac{L_{\tau}-1 / 3 L_{x+y+z}}{L_{\tau}+1 / 3 L_{x+y+z}}
$$

where, for example, $L_{\tau}=N_{\tau} \cdot a$ and $N_{\tau}$ is the number of links belonging to the percolating monopole trajectories and looking in the (Euclidean) time direction. A similar parameter can be introduced for magnetic strings, in terms of the total areas occupied by plaquettes belonging to the surfaces and oriented in various ways.

At low temperatures the asymmetry $v=0$. At temperatures close to the temperature of the phase transition $T_{c}$ there appears a non-zero $v$. And at about $T \sim 1.5 T_{c}$ the time-oriented links dominate. 


\section{Stringy models for Yang-Mills theories}

\subsection{Geometry of extra dimensions}

A dual, or stringy description of Yang-Mills theories has not been yet constructed. However, there exists a model [12] which belongs to the same universality class as the large- $N_{c}$ Yang-Mills theories in the far infrared.

The corresponding geometry involves ten dimensions, where the number of ten is fixed by the requirement to have a consistent string theory at short distances. For our purposes, we concentrate on the standard four dimensions, fifth dimension, denoted here as $u$ and one extra compact coordinate, $x_{4}$. The fifth, mass scale-conjugated dimension is common to all dual models. The limit $u \rightarrow \infty$ means short distances in our world while finite values of $u$ correspond to poorer resolution in our world, or moving towards the infrared. The $x_{4}$ coordinate is specific for the universality class of the Yang-Mills theories [12]. At temperature $T=0$ the metric is:

$$
\begin{array}{r}
d s^{2}=\left(\frac{u}{R}\right)^{3 / 2}\left(-d t^{2}+\delta_{i j} d x^{i} d x^{j}+f(u) d x_{4}^{2}\right)+\left(\frac{u}{R}\right)^{3 / 2}\left(\frac{d u^{2}}{f(u)}+u^{2} d \Omega_{4}^{2}\right) \\
f(u)=1-\left(\frac{u_{\Lambda}}{u}\right)^{3}, \quad x_{4} \sim x_{4}+\beta_{4}, \quad \beta_{4}=\frac{4 \pi}{3}\left(\frac{R^{3}}{u_{\Lambda}}\right),
\end{array}
$$

where $R$ is a constant related to the Y-M coupling constant. Note the existence of a horizon at $u=u_{\Lambda}$ which is crucial to ensure confinement. Moreover, the $x_{4}$ coordinate is related to the topological charge. Namely, if a defect is wrapped once over the $x_{4}$ circle, it has a unit topological charge. Wrapping in the opposite direction brings a minus sign for the topological charge. Extra compact dimensions, associated with the unit sphere $\Omega_{4}$ are relevant to baryons and do not concern us here.

At finite Euclidean temperatures, there are two compact coordinates, $x_{4}$ and the Euclidean time, $\tau$. The deconfinement phase transition is identified as the Hawking-Page transition which interchanges the geometries in the $\left(x_{4}, u\right)$ coordinates and in the $(\tau, u)$ coordinates [13]. Namely, at low temperatures the geometry in the $\left(u, x_{4}\right)$ coordinates is cigar-shaped since the radius of the $x_{4}$-coordinate tends to zero at the horizon:

$$
R_{\tau}(u)=\frac{1}{2 \pi T}, R_{x_{4}}\left(u_{\Lambda}\right)=0 ; T<T_{c} .
$$

On the other hand, the radius of the Euclidean time direction is independent of $u$.

At temperatures above the phase transition the geometry of the two compact coordinates is interchanged so that:

$$
R_{\tau}\left(u_{T}\right)=0, R_{x_{4}}(u)=\text { const } ; T>T_{c},
$$

where $u_{T}$ is the position of the temperature-related horizon. The phase transition takes place at the temperature where the two radii, namely, $R_{\tau} \equiv 1 / 2 \pi T$ and the value of $\beta_{4} / 2 \pi$, become equal to each other.

\subsection{Low-dimensional defects}

What kind of physics could the model (3.1) describe? At short distances, or at $u \rightarrow \infty$, the model has five dimensions, instead of four and is apparently not relevant. The model can be valid only at distances $x \gg \beta_{4} \sim \Lambda_{Q C D}^{-1}$, or at values of $u$ close to the horizon. 
Thus, at $T>T_{c}$ we are left with hydrodynamics of the Yang-Mills plasma as potential application of the model (3.1). At temperature $T=0$ one could apply the model to the vacuum which is - in our context - nothing else but the theory of low-dimensional defects, like monopoles and magnetic strings. Moreover, in all the cases, it is more appropriate to talk about the non-perturbative components (say, of the same plasma) since the perturbative contributions come from short distances and cannot be described within the model (3.1).

Concerning the notion of defects, the Polyakov line [14] can be considered as a prototype of such defects. It is defined in the Euclidean space as

$$
L(\mathbf{x}) \equiv \frac{1}{N_{c}} \operatorname{Tr} P \exp \left(-i \int_{0}^{1 / T} A_{0}(\mathbf{x}, \tau) d \tau\right),
$$

where $\tau$ is the Euclidean (periodic) time, $A_{0}$ is the gluon field. Note that the Polyakov line depends only on the spatial coordinates since one integrates over the time direction along the line. From the point of view of the $3 \mathrm{~d}$ theory the Polyakov line (3.4) is a point-like defect. It was argued first in Ref. [14] that actually this defect might represent a dynamical degree of freedom at $T>T_{c}$ which is to be added to the Hamiltonian of the Yang-Mills theories by hand. Later, there appeared many models which try to make this picture more precise, see [15] and references therein.

In the models, like (3.1) there are many more defects. Indeed, according to the theory there exist D0, D2, D4 branes, for details and incomplete list see, in particular, [7]. According to the general rules, the probability to find such defects is exponentially small in the limit of large $N_{c}$,

$$
S_{\text {defect }} \sim N_{c} \cdot T \cdot(\text { Volume })_{\text {defect }},
$$

where $T$ is a generalized tension and $(\text { Volume })_{\text {defect }}$ is the volume occupied by the defect. Such defects are not significant dynamically. However, there are cases when the volume of the defect vanishes,

$$
(\text { Volume })_{\text {defect }}=0, \quad S_{\text {defect }}=0
$$

in the classical approximation. Such defects can become dynamical and, in particular, might correspond to the percolating monopoles or magnetic strings.

\subsection{Magnetic strings in dual models}

Note that all the branes wrapped around the $x_{4}$ compact dimension at temperature $T=0$ satisfy the condition (3.5) and can become dynamical in the infrared limit. In particular, consider D2 branes which are wrapped around the $x_{4}$ coordinates and extended in two other directions of the Euclidean $4 \mathrm{~d}$ space. Then at zero temperature the action associated with such defects is vanishing in the infrared:

$$
S_{D 2 \text { branes }}(u) \sim R_{x_{4}}(u) \cdot(\text { Area })_{4 d},
$$

where $(\text { Area })_{4 d}$ is the area swept by the brane in the Euclidean $4 \mathrm{~d}$. Because of (3.2) the brane action vanishes for the branes living on the horizon, $u \rightarrow u_{\Lambda}$.

Let us interpret this observation. First of all, "living on the horizon " means large distances, or the infrared limit. In field theoretic language, for example, instantons have action vanishing in the infrared. In other words, they are not suppressed by the action and populate the vacuum. Now, a similar picture holds for the 2D branes wrapped around the $x_{4}$ circle. Thus, in $4 \mathrm{~d}$ these branes 
could look as percolating $2 \mathrm{~d}$ surfaces. This picture fits the lattice magnetic strings described above [6].

Furthermore, the surfaces should be topologically charged since the D2 branes are wrapped around the $x_{4}$ direction. This prediction also fits the lattice data which indicate that the topological fermionic modes are indeed strongly correlated with the magnetic strings.

What happens at $T=T_{c}$ according to the dual model? As is mentioned above, see (3.3), the radius of the (Euclidean) $\tau$-circle is now vanishing at the horizon while the radius of the $x_{4}$ dimension does not depend on $u$. Thus, wrapping around the $x_{4}$ circle does not ensure a vanishing action any longer. However, the D2 branes can still be dynamical degrees of freedom if they are wrapped around the $\tau$-circle. In the geometric language wrapping around the time direction means that the defects become time oriented.

If a surface becomes parallel to the Euclidean time, then the time dependence is trivial and one can concentrate on a $3 \mathrm{~d}$ time slice of the lattice. Moreover, the intersection of the surface with the time slice is a line. And in the language of the quantum geometry lines represent particles in any number of dimensions. The infinite percolating cluster of surfaces at $T>T_{c}$ is becoming an infinite percolating cluster of trajectories in $3 \mathrm{~d}$.

\section{Towards applications}

\subsection{Massless modes, stringy $U(1)$ symmetries}

Since we are discussing distances much larger than $\Lambda_{Q C D}^{-1}$ we are in fact interested in gapless excitations. Moreover, $3 \mathrm{~d}$ massless scalars are known to be a signature of superfluidity. Thus, if we find such a scalar in the realistic set up of the Yang-Mills theories, there are good chances that the quark-gluon plasma contains a superfluid component. What are the mechanisms of generating massless scalars? First, Goldstone bosons appear in case of spontaneous symmetry breaking, or non-vanishing vacuum expectation value of a complex field. Massless modes can also be related to defects with finite action, as zero modes. Both mechanisms could be relevant to the Yang-Mills plasma.

In this context, the central point is that there exist specific stringy $U(1)$ symmetries associated with compact dimensions. As an example, consider temperature $T=0$ and the compact $x_{4}$ coordinate. Then the states of strings wrapped around this coordinate would have a $U(1)$ charge which is nothing else but the wrapping number

$$
Q=n_{w r} .
$$

Moreover, since the $x_{4}$ direction is associated with the topological charge, the wrapping number $n_{w r}$ fixes also the topological charge of the corresponding state. Naively, the corresponding current would look like

$$
J_{\mu}=q_{t o p} \Phi_{1}^{*} \partial_{\mu} \Phi_{1}+2 q_{t o p} \Phi_{2}^{*} \partial_{\mu} \Phi_{2}+\ldots
$$

where $\Phi_{1,2}$ are wave functions of the state with $n_{w r}=1,2$ and $q_{t o p}$ is a unit charge. Generically, all such states are heavy and cannot be consistently used in our model.

However, the cigar-shaped geometry in the coordinates $\left(u, x_{4}\right)$ suggests that the corresponding $U(1)$ symmetry can well be spontaneously broken. Then there appears a massless Goldstone, $\phi_{\theta}$, 
and the effective Lagrangian for this field would be axion-like:

$$
L\left(\left(\phi_{\theta}\right)=1 / 2\left(\partial_{\mu} \phi_{\theta}\right)^{2}+f_{\theta} \partial_{\mu} \phi_{\theta} K_{\mu}+\ldots,\right.
$$

where the dots involve states with masses of order $\Lambda_{Q C D}$ and should be neglected in our approximation and $K_{\mu}$ is the topological Chern-Simons current. The current (4.1)becomes in the approximation of light states $J_{\mu} \sim f_{\theta}^{2} \partial_{\mu} \theta+K_{\mu}$.

Another $U(1)$ symmetry, much more discussed in the literature ${ }^{2}$ is the topological symmetry associated with the wrapping around the compact Euclidean type. In the deconfining phase, $T>T_{C}$, the cigar-shape geometry is in the coordinates $(u, \tau)$ and this symmetry could well be spontaneously broken. Denote the corresponding Goldstone as $\phi_{\text {thermal }}$. Then the corresponding $U(1)$ current would look as

$$
J_{i}=f_{\text {th }}^{2} \partial_{i} \phi_{\text {thermal }}+\ldots, i=1,2,3,
$$

where we again do not specify the contribution of massive states. If $\mathrm{Eq}(4.3)$ is true then it signals superfluidity, for details and references see [18],

\subsection{Massless states as vibration of branes}

As we discussed above, the non-perturbative physics in the Euclidean space becomes static at $T>T_{c}$. At large number of colors, the first-order phase transition can be considered as a change in the number of dimensions of non-perturbative physics [16]. At $N_{c}=3$ the transition is somewhat smoother.

Thus, we can consider the non-perturbative defects as living on a $3 \mathrm{~d}$ subspace, embedded into the $4 \mathrm{~d}$ Euclidean space. Then the classical action associated with the $3 \mathrm{~d}$ brane is

$$
S_{3 d, \text { classical }}=-T \cdot \int d^{3} x .
$$

Let us emphasize the unconventional minus sign in right-hand side. The common expression would of course be with the plus sign, and $e^{-S_{\text {classical }}}$ would give the probability to excite the defect. However, in our case the nonperturbative defects lower the energy of the vacuum. They are always present in the $3 \mathrm{~d}$ space. In other words, the cosmological constant associated with the nonperturbative effects corresponds to a negative energy, as is discussed first in connection with the gluon condensate in QCD [17]. More formally, non-perturbative contribution to the vacuum energy is to be understood as the difference between the energy of the true and perturbative vacuum. This difference is negative.

In the quasi-classical approximation the action becomes

$$
S_{3 d, \text { quasi-classical }}=-T \cdot \int d^{3} x \sqrt{1+\left(\partial_{i} \phi\right)^{2}},
$$

where $\phi$ is a massless $3 \mathrm{~d}$ state, the physical meaning of $\phi$ being $\phi=\tau$ where $\tau$ is the Euclidean time. The massless particle describes vibrations of the $3 \mathrm{~d}$ brane in the Euclidean time direction. Continuing (4.5) to the Minkowski space we change the overall sign and replace $\tau \rightarrow i \tau$ to get:

$$
S_{3 d, \text { Minkowski }}=+T \cdot \int d^{3} x \sqrt{1-\left(\partial_{i} \phi\right)^{2}} .
$$

Eq. (4.6) can serve as a starting point for applications.

\footnotetext{
${ }^{2}$ for an incomplete list see [19].
} 


\subsection{Exotic liquid}

Static physics is the same in the Euclidean and Minkowski spaces. Thus, we could apply (4.6) directly to Yang-Mills plasma. However, the applications become much richer if a relativistic generalization is known. One can argue that the exotic liquid found in [20] does provide us with such a generalization. Let us describe briefly the results of [20]. One considers the action of a $4 \mathrm{~d}$ scalar field $\phi$

$$
S=T \int d^{4} x \sqrt{-\gamma} \sqrt{-(\partial \phi)^{2}}
$$

where $\gamma$ is the determinant of the metric tensor $\gamma_{a b}(a, b=0,1,2,3)$ defined as:

$$
\gamma_{a b} d x^{a} d x^{b}=-r_{c} d \tau^{2}+d x_{i} d x^{i}
$$

where $r_{c}$ is arbitrary at the moment. The central point is to identify normalized gradient of $\phi$ as the 4-velocity of an ideal liquid $u_{a}$ :

$$
u_{a}=\partial_{a} \phi / \sqrt{X}, X \equiv-(\partial \phi)^{2} \equiv\left(\partial_{0} \phi\right)^{2}-\left(\partial_{i} \phi\right)^{2} .
$$

Another crucial point is that the equilibrium solution is

$$
\phi_{\text {equilibrium }}=t .
$$

Now, we are in position to establish a relation to our model (4.6). Indeed, considering static case $\phi=\phi_{\text {equilibrium }}+\delta \phi(\mathbf{r})$ we find that the action (4.7) for the $3 \mathrm{~d}$ brane coincides with (4.6). However, the integration measure over the the time $t$ in (4.7) is far from being trivial and reflects certain dynamical assumptions, for the background see [21]. Namely, the liquid (4.7) is dual to Rindler space with one extra dimension. Note that near the horizon $r_{c} \rightarrow 0$ and the $4 \mathrm{~d}$ space is becoming $3 \mathrm{~d}$ space, as it should be on the horizon. From our perspective, the crucial point is that as far as the Rindler horizon approximates the (temperature) horizon of the model (3.1) we can use results of [20] to predict the properties of the non-perturbative component of the Yang-Mills plasma.

\subsection{Properties of the Yang-Mills plasma}

The most unusual property of the liquid (4.7) is that in equilibrium it has vanishing energy and non-vanishing pressure:

$$
\left(T_{a b}\right)_{\text {equilibrium }}=(0, p, p, p), \quad p=1 / \sqrt{r_{c}} .
$$

The physical picture behind this observation can be readily understood in terms of the defects we are considering. Indeed, say the D2 magnetic vortices percolate in $3 \mathrm{~d}$ and in this way create pressure (which is a cosmological constant in the $3 \mathrm{~d}$ language). Their time dependence, on the other hand, is trivial. Note that pressure $p \sim r_{c}^{-1 / 2}$ and blows up at the horizon. In reality, this behaviour should be tempered (because approximations may fail).

As is emphasized in [23] the property (4.10) echoes properties of the liquid living on the stretched horizon of a black hole. Also, there are properties of the liquid which are similar to a superfluid. In particular, in the ideal-liquid approximation the entropy $s=0$ while after the inclusion of dissipation effects the ratio of the viscosity to the entropy takes [20] the lowest value 
possible, $\eta / s=1 / 4 \pi$. Also the 4 -velocity of the liquid, see (4.9), is rotationless, the same as for a superfluid.

On the lattice, one can measure separately the contribution of magnetic strings into the equation of state of the plasma. In particular, the contribution of magnetic strings to the trace of energy momentum tensor

$$
T_{\mu}^{\mu}=\varepsilon-3 p
$$

was measured on the lattice [22]. It turned out that

$$
\left(T_{\mu}^{\mu}\right)_{\text {strings }}<0
$$

and very large numerically. Qualitatively, this result is in very nice agreement with the properties of the non-perturbative component of the plasma we are discussing. We cannot rule out, of course, that the coincidence could be accidental.

\section{Conclusions. From confinement to superfluidity?}

We have argued that there is ample evidence on the lattice in favor of existence of scalar condensates both at temperature $T=0$ where the non-perturbative physics is four-dimensional and at temperatures above the phase transition, $T>T_{c}$ where non-perturbative physics becomes three-dimensional. The dual model (3.1) which is in the same universality class as the Yang-Mills theories in deep infrared region does suggest that there are stringy $U(1)$ symmetries associated with wrapping around compact (Euclidean) coordinates. Moreover, the cigar shape geometry in the fifth dimension suggests spontaneous breaking of the corresponding $U(1)$ symmetry, resulting in the formation of a scalar condensate.

In case of $3 \mathrm{~d}$ physics, that is at $T>T_{c}$, it turned out to be possible to predict properties of the Yang-Mills plasma which are both highly non-trivial and are in no contradiction with the lattice data. At this point we used the results of Ref. [20] where an exotic liquid was found (motivated by absolutely different considerations).

We have not discussed in any detail the low temperature physics. However, it looks probable that the vacuum expectation of the monopole field, observed on the lattice, signifies just the spontaneous breaking of the stringy $U(1)$ associated with the topological charge (or extra $x_{4}$-coordinate), discussed above. Moreover the vanishing of the residue for exchanges of the corresponding Goldstone particle might fit the vanishing of the monopole condensate in the limit $a \rightarrow 0$, discussed in Sect. 2.2.

\section{Acknowledgments}

The work of VIZ is partially supported by grants Leading Scientific Schools NSh-6260.2010.2, RFBR -11-02-01227-à and Federal Special-Purpose Programme 'Cadres' of the Russian Ministry of Science and Education. 


\section{References}

[1] A. M. Polyakov, Phys. Lett. B59 82 (1975);

A. M. Polyakov, Nucl. Phys. B120 (1977) 429.

[2] J. Greensite, Prog. Part. Nucl. Phys. 51 (2003) 1. [arXiv:hep-lat/0301023].

[3] A. M. Polyakov, "Gauge fields and Strings," Harwood Academic Publishers (1987).

[4] V.I. Zakharov, in Sense of Beauty in Physics: Miniconference in Honor of Adriano Di Giacomo on his 70th Birthday, Pisa, Italy, 26-27 Jan 2006, [arXiv:hep-ph/0602141].

[5] V.I. Zakharov, Braz. J. Phys. 37 (2007) 65, [arXiv:hep-ph/0612342];

V.I. Zakharov, AIP Conf.Proc. 756182 (2005), [arXiv:hep-ph/0501011].

[6] A. Gorsky, V. Zakharov, Phys. Rev. D77 (2008) 045017, arXiv:0707.1284 [hep-th].

[7] A. S. Gorsky, V. I. Zakharov, A. R. Zhitnitsky, Phys. Rev. D79 (2009) 106003, arXiv:0902.1842 [hep-ph].

[8] D. Teaney, Prog. Part. Nucl. Phys. 62 (2009) 451.

[9] V.G. Bornyakov et al., Phys. Lett. B537 (2002) 291, [arXiv:hep-lat/0103032];

V.G. Bornyakov, P.Yu. Boyko, M.I. Polikarpov, V.I. Zakharov, Nucl. Phys. B672 (2003) 222,

[arXiv:hep-lat/0305021].

[10] M.N. Chernodub, V.I. Zakharov, Nucl. Phys. B669 (2003)233, [arXiv:hep-th/0211267].

[11] F.V. Gubarev, A.V. Kovalenko, M.I. Polikarpov, S.N. Syritsyn, V.I. Zakharov, Phys. Lett. B574 (2003) 136, [arXiv:hep-lat/0212003].

[12] E. Witten, Adv. Theor. Math. Phys. 2 (1998) 505, [arXiv:hep-th/9803131].

[13] O. Aharony, J. Sonnenschein, Sh. Yankielowicz, Annals Phys. 322 (2007) 1420, [arXiv:hep-th/0604161].

[14] A. M. Polyakov, Phys. Lett. B72 (1978) 477.

[15] A. Dumitru, Y. Guo, Y. Hidaka, Ch. P. Korthals Altes, R. D. Pisarski, Phys. Rev. D83 (2011) 034022. arXiv:1011.3820 [hep-ph].

[16] M.N. Chernodub, A. Nakamura, V.I. Zakharov, Proc. Steklov Inst. Math. 272 (2011) 75, arXiv:0904.0946 [hep-ph].

[17] M.A. Shifman, A.I. Vainshtein, V.I. Zakharov, Nucl. Phys. B147 (1979) 385.

[18] H. Verschelde, and V.I. Zakharov, arXiv:1012.4821 [hep-th]; M.N. Chernodub, H. Verschelde, V.I. Zakharov, Nucl. Phys. Proc. Suppl. 207-208 (2010) 325, arXiv:1007.1879 [hep-ph].

[19] B. Sathiapalan, Phys. Rev. D35 (1988) 3277;

Ya. I. Kogan, JETP Lett. 45 (1987) 709;

J. J. Atick, and E. Witten, Nucl. Phys. B310 (1988) 291;

A. Adams, X. Liu, J. McGreevy, A. Saltman, E. Silverstein, JHEP 0510 (2005) 033, [arXiv:hep-th/0502021];

G. T. Horowitz, JHEP 0508 (2005) 091, e-Print: hep-th/0506166;

G. T. Horowitz, E. Silverstein, Phys. Rev. D73 (2006) 064016, [arXiv:hep-th/0601032];

M. Kruczenski, and A. Lawrence, JHEP 0607 (2006) 031, [arXiv:hep-th/0508148].

[20] G. Compere, P. McFadden, K. Skenderis, M. Taylor, arXiv:1103.3022 [hep-th]. 
[21] I. Bredberg, C. Keeler, V. Lysov, A. Strominger, JHEP 1103 (2011) 141, arXiv:1006.1902 [hep-th], arXiv:1101.2451 [hep-th].

[22] M.N. Chernodub, A. Nakamura, V.I. Zakharov, Phys. Rev. D78 (2008) 074021, arXiv:0807.5012 [hep-lat].

[23] H. Verschelde, and V.I. Zakharov, arXiv:1106.4154 [hep-th] 